\title{
Amino Acid Profile in 18 Patients with Rheumatic Diseases Treated with Glucocorticoids and BCAAs
}

\author{
Noritada Yoshikawa $^{1,2}$, Motohisa Yamamoto ${ }^{1, *}$, Akiko KurIBARA-SOUTA ${ }^{1}$, \\ Masaaki UEHARA $^{1}$, Hiroki YAMAZAKI ${ }^{1}$ and Hirotoshi TANAKA ${ }^{1,2}$ \\ ${ }^{1}$ Department of Rheumatology and Allergy, IMSUT Hospital, The Institute of Medical Science, The Univer- \\ sity of Tokyo, 4-6-1, Shirokanedai, Minato-ku, Tokyo 108-8639, Japan \\ ${ }^{2}$ Division of Rheumatology, Center for Antibody and Vaccine Therapy, IMSUT Hospital, The Institute of \\ Medical Science, The University of Tokyo, 4-6-1, Shirokanedai, Minato-ku, Tokyo 108-8639, Japan
}

(Received January 22, 2021)

\begin{abstract}
Summary The administration of glucocorticoids to patients with rheumatic diseases often results in glucocorticoid-induced myopathy. We previously found that administration of branched-chain amino acids (BCAA) to such patients improves the loss of skeletal muscle, however, their individual differences were often observed. The present study, therefore, aims to identify specific parameters associated with BCAA-induced increases in skeletal muscle mass. Eighteen patients with rheumatic diseases treated with prednisolone were randomly assigned to receive additional BCAAs for $12 \mathrm{wk}$. Serum biochemistry, plasma fibroblast growth factor (FGF) 19 and 21, and plasma and urinary amino acid concentrations were assessed. The relationship between these parameters and the cross-sectional area (CSA) of the biceps femoris (slow-twitch muscle) and rectus femoris (fast-twitch muscle) was assessed using computed tomography. BCAA supplementation increased serum levels of creatinine and albumin and decreased ammonia and urinary 3-methylhistidine levels. With or without BCAA supplementation, each plasma amino acid concentration decreased during the study period, but the decrease was lower in patients receiving BCAA. Interestingly, a positive correlation was observed between plasma isoleucine, aspartate, and glutamate concentrations and improvement in the biceps femoris muscle atrophy. Plasma amino acid concentrations in patients with rheumatic diseases treated with glucocorticoids decreased despite tapering the dose of glucocorticoids, with a smaller decrease in the BCAAtreated group. Plasma BCAA, aspartic acid, and glutamate concentrations correlated positively with the rate of improvement in biceps femoris muscle atrophy, suggesting that these amino acids are associated with the BCAA-induced increase in muscle mass.
\end{abstract}

Key Words branched-chain amino acid, glucocorticoid, muscle atrophy, myopathy, sarcopenia

Skeletal muscle atrophy is a complication of many conditions and treatments, including malignancies, heart failure, renal failure, lung disease, chronic inflammatory diseases, glucocorticoid (GC) treatment, and aging $(1,2)$. Ameliorating skeletal muscle atrophy can improve the quality of life of a patient, regardless of the underlying disease status $(1,2)$. Therefore, identifying the mechanisms underlying skeletal muscle atrophy and developing new strategies to prevent it could have a significant impact on these patients. Although GCs are important in the treatment of many inflammatory diseases, long-term GC treatment can induce skeletal muscle atrophy $(3,4)$. We have shown that GCs decrease muscle protein synthesis while simultaneously promoting protein degradation by inhibiting the mammalian mechanistic target of rapamycin (mTOR)/S6 kinase 1 pathway and regulating the expression of various muscle-atrophy-related genes (5-7). mTOR-activating

\footnotetext{
*To whom correspondence should be addressed.

E-mail: mocha@ims.u-tokyo.ac.jp
}

branched-chain amino acids (BCAA) are potential therapeutic candidates for reducing GC-induced myopathy (5). Thus, we previously conducted a translational study to examine the clinical effects of BCAA supplementation on skeletal muscle mass, strength, and function in patients with rheumatic diseases treated with GCs (8). Initially, we expected that GC-induced myopathy would improve in all cases, but in fact, we observed individual differences in the degree of improvement even in the BCAA-treated patients. To identify specific factors involved in this improvement in muscle atrophy, this study aims to determine whether plasma and urinary amino acid concentrations are associated with improved muscle atrophy.

\section{MATERIALS AND METHODS}

Subjects. The study cohort included patients with rheumatic disorders, age $\geq 20 \mathrm{y}$, taking or planning to take prednisolone $(\geq 10 \mathrm{mg} / \mathrm{d})$ for at least $3 \mathrm{mo}$, and who were regularly followed in the Department of Rheumatology and Allergy at IMSUT Hospital, Institute 
of Medical Science, The University of Tokyo (7). Patients were diagnosed by three rheumatologists who were certified by the Japan College of Rheumatology board in our department. Exclusion criteria included uncontrolled diabetes mellitus (HbAlc $\geq 8.4 \%$ ); significant heart, kidney, liver, blood, or respiratory disease; severe infectious disease; active cancer; metabolic disorders of amino acids; hypoalbuminemia; and pregnant or lactating women. Each patient was randomly assigned to one of two groups to receive BCAA supplementation or not (BCAA + or $\mathrm{BCAA}-$, respectively). Nine patients in each group completed the study and were included in the data analysis. This study was registered at the University Hospital Medical Information Network Clinical Trials Registry (UMINO00006972, 29/12/2011) and approved by the Ethics Committee of the Institute of Medical Science, The University of Tokyo (No. 23-5, 24-11, 25-19, 26-17). All patients were informed of the purpose of the study and provided written consent.

Trial design (7). This phase I-II, open label, randomized, parallel group clinical trial was performed in 18 subjects on an ordinary diet with or without additional BCAA supplementation. BCAA supplementation was administered orally with a commercially available concentrated BCAA drink (Amino-value $\mathrm{CONC}^{\circledR}$, Otsuka Pharmaceutical, Co., Ltd., Tokyo, Japan). The content of 100-mL of BCAA solution was as follows: energy, $58 \mathrm{kcal}$; carbohydrates, $12.1 \mathrm{~g}$; valine, $500 \mathrm{mg}$; leucine, 1,000 mg; isoleucine, $500 \mathrm{mg}$ (BCAA total, $2 \mathrm{~g}$ ). $\mathrm{BCAA}+$ patients were instructed to take two bottles of Amino-value $\mathrm{CONC}^{\circledR}$ after each meal, three times a day (12 $\mathrm{g}$ bolus supplementation of BCAA per day) for $12 \mathrm{wk}$. Patients recorded their intake in a log book that was verified by research personnel at regularly scheduled visits. Computed tomography (CT) scans were performed within $2 \mathrm{wk}$ before and $12 \mathrm{wk}$ after the trial. Physical examination, blood and urine analysis, and assessment of disease activity and BCAA safety were performed at every visit. The participants were advised to maintain an ordinary lifestyle, neither taking commercially available BCAA-containing food nor performing particular exercise for muscle recovery. We monitored the compliance and behavior of the patients using a detailed questionnaire on daily living. The overall compliance of BCAA ingestion was 93\%. This study was conducted from May 2012 to January 2015.

CT acquisition and analysis (7). To evaluate the skeletal muscle cross-sectional area (CSA), a transverse CT scan of the thigh halfway between the head of the femur and the knee joint space was performed using a LightSpeed Ultra CT scanner (GE Healthcare Japan, Tokyo, Japan). All CT data were analyzed using ImageJ software from the National Institutes of Health with thresholding methods (9). We obtained the bilateral CSA $\left(\mathrm{mm}^{2}\right)$ of the mid-thigh muscle by measuring the area with attenuation values between -29 and +150 Hounsfield units with two blinded investigators and corrections for body height. To measure CSAs, manual tracing was performed to separate the fascia of rectus femoris and biceps femoris.
Laboratory evaluation and amino acid analysis. Blood and urine samples were collected in the morning after an overnight fast. A part of plasma, serum, and urine samples were snap frozen in liquid nitrogen and subsequently stored at $-80^{\circ} \mathrm{C}$, until used for further analyses. Complete blood counts and serum and urinary biochemical analyses were performed at the clinical laboratory in our hospital using a Sysmex XN-1000 Hematology Analyzer (Sysmex Corporation, Kobe, Japan) and LABOSPECT 006 Hematology Analyzer (Hitachi HighTech Corporation, Tokyo, Japan), respectively. Serum insulin and free fatty acids were measured by chemiluminescent enzyme immunoassay (ELISA) in a medical laboratory (SRL, Inc., Tokyo, Japan). Plasma levels of fibroblast growth factor (FGF) 19 and FGF21 were quantified using the Human FGF19 and FGF21 Quantikine ELISA Kit (DF1900 and DF2100, respectively; R\&D systems, Minneapolis, MN) and iMark microplate absorbance reader (Bio-Rad, Hercules, CA, USA) according to the manufacturer's instructions. The levels of plasma and urine amino acids were analyzed using a high-performance liquid chromatography/electrospray mass spectrometry system consisting of a LaChrom Elite L-2000 series liquid chromatography system, an L-2200 auto-sampler, two L-2100 pumps, an L-2130 pump, and an L-2300 column oven (Hitachi High-Tech) at SRL, Inc.

Ethical considerations. This study was registered at UMIN Clinical Trials Registry (UMINO00006972), 29/12/2011, and approved by the Ethics Committee of the Institute of Medical Science, The University of Tokyo (No. 23-5, 24-11, 25-19, 26-17). Written informed consent was obtained from all patients, and as well as of their rights and potential risks of the study in accordance with the Helsinki Declaration.

Statistical analysis. Statistical comparisons of mean values were obtained using Student's $t$-test. Categorical variables were compared between the $\mathrm{BCAA}+$ and BCAA - groups using the chi-square test. Pearson product-moment correlation analysis was used to investigate the correlation coefficients for each parameter. $p<0.05$ was considered significant. Data are presented as the mean \pm standard error of the mean, as indicated.

\section{RESULTS}

\section{Patient profiles and thigh muscle assessment}

As we previously reported (7), the cohort included 18 subjects (age range, 20-76 y) diagnosed with a variety of rheumatic diseases. Five subjects had systemic lupus erythematosus and four had polymyalgia rheumatica. Adult-onset Still's disease, immunoglobulin (Ig)G4-related disease, and anti-neutrophil cytoplasmic antibody (ANCA)-related vasculitis were present in two patients each, and giant cell arteritis, rheumatoid arthritis, and myositis were present in one patient each. No difference in sex ratio, age at the start of the study, body size, or muscle strength was observed between the BCAA - and $\mathrm{BCAA}+$ groups. With regard to GC treatment, no significant differences were observed between the two groups with respect to previous maximum dose, total dose in 
Table 1. Biochemical profiles of the participants.

\begin{tabular}{|c|c|c|c|c|c|c|c|}
\hline & \multirow{2}{*}{ Standard values } & \multicolumn{3}{|c|}{$\mathrm{BCAA-}$} & \multicolumn{3}{|c|}{$\mathrm{BCAA}+$} \\
\hline & & $0 \mathrm{wk}$ & $12 \mathrm{wk}$ & $p$-Value & $0 \mathrm{wk}$ & $12 \mathrm{wk}$ & $p$-Value \\
\hline AST & 13-30 U/L & $19.4 \pm 1.6$ & $19.7 \pm 1.1$ & 0.850 & $14.8 \pm 1.5$ & $22.0 \pm 2.4$ & $0.007^{*}$ \\
\hline ALT & $10-42 \mathrm{U} / \mathrm{L}$ & $27.9 \pm 4.7$ & $21.4 \pm 2.3$ & 0.198 & $14.8 \pm 1.5$ & $26.4 \pm 5.7$ & 0.095 \\
\hline $\mathrm{LDH}$ & $124-222 \mathrm{U} / \mathrm{L}$ & $208.6 \pm 14.5$ & $253.6 \pm 29.7$ & 0.147 & $214.1 \pm 16.1$ & $226.8 \pm 16.5$ & 0.194 \\
\hline$\gamma \mathrm{GTP}$ & 13-64 U/L & $33.9 \pm 5.3$ & $31.4 \pm 5.6$ & 0.370 & $42.8 \pm 11.1$ & $42.4 \pm 5.5$ & 0.974 \\
\hline BUN & $8-20 \mathrm{mg} / \mathrm{dL}$ & $16.9 \pm 1.9$ & $14.1 \pm 1.6$ & $0.013^{*}$ & $14.0 \pm 1.4$ & $14.7 \pm 2.3$ & 0.611 \\
\hline CRE & $0.65-1.07 \mathrm{mg} / \mathrm{dL}$ & $0.64 \pm 0.05$ & $0.63 \pm 0.04$ & 0.714 & $0.65 \pm 0.05$ & $0.71 \pm 0.06$ & $0.006^{*}$ \\
\hline Alb & $3.8-5.3 \mathrm{~g} / \mathrm{dL}$ & $3.7 \pm 0.1$ & $4.1 \pm 0.1$ & $0.033^{*}$ & $3.62 \pm 0.1$ & $4.00 \pm 0.10$ & $0.002 *$ \\
\hline CPK & $45-287 \mathrm{U} / \mathrm{L}$ & $53.7 \pm 21.4$ & $67.0 \pm 19.5$ & 0.271 & $33.11 \pm 6.6$ & $51.00 \pm 8.8$ & $0.024^{*}$ \\
\hline $\mathrm{NH}_{3}$ & $12-66 \mu \mathrm{g} / \mathrm{dL}$ & $37.4 \pm 6.4$ & $24.6 \pm 1.5$ & 0.090 & $51.2 \pm 5.3$ & $33.1 \pm 3.4$ & $0.001^{*}$ \\
\hline CRP & $<0.14 \mathrm{mg} / \mathrm{dL}$ & $0.10 \pm 0.05$ & $0.02 \pm 0.01$ & 0.161 & $0.03 \pm 0.01$ & $0.02 \pm 0.00$ & 0.081 \\
\hline Glu & $73-109 \mathrm{mg} / \mathrm{dL}$ & $98.4 \pm 4.5$ & $97.8 \pm 3.5$ & 0.905 & $102.3 \pm 7.9$ & $105.3 \pm 4.2$ & 0.724 \\
\hline $\mathrm{HbA1c}$ & $4.9-6.0 \%$ & $5.7 \pm 0.1$ & $5.9 \pm 0.2$ & 0.332 & $5.9 \pm 0.1$ & $5.8 \pm 0.1$ & 0.181 \\
\hline Insulin & $1.84-12.2 \mu \mathrm{IU} / \mathrm{mL}$ & $14.1 \pm 2.8$ & $8.6 \pm 1.8$ & 0.129 & $9.9 \pm 1.6$ & $10.9 \pm 2.1$ & 0.610 \\
\hline HOMA-IR & $<1.6$ & $3.45 \pm 0.71$ & $2.03 \pm 0.40$ & 0.135 & $2.55 \pm 0.48$ & $2.84 \pm 0.56$ & 0.626 \\
\hline TCho & $150-219 \mathrm{mg} / \mathrm{dL}$ & $213.9 \pm 16.5$ & $208.0 \pm 13.8$ & 0.418 & $244.9 \pm 13.6$ & $226.4 \pm 7.8$ & 0.291 \\
\hline TG & $50-149 \mathrm{mg} / \mathrm{dL}$ & $131.4 \pm 10.1$ & $114.6 \pm 18.3$ & 0.370 & $134.6 \pm 18.1$ & $118.1 \pm 19.2$ & 0.330 \\
\hline FFA & $140-850 \mu \mathrm{Eq} / \mathrm{L}$ & $366.7 \pm 73.4$ & $637.0 \pm 140.9$ & $0.039^{*}$ & $505.8 \pm 69.0$ & $565.2 \pm 73.8$ & 0.458 \\
\hline
\end{tabular}

Alb, albumin; ALT, alanine aminotransferase; AST, aspartate aminotransferase; BUN, blood urea nitrogen; CPK, creatine phosphokinase; CRE, creatinine; CRP, C-reactive protein; FFA, free fatty acids; $\gamma$ GTP, $\gamma$-glutamyl transpeptidase; Glu, glucose; HbAlc, hemoglobin A1C; HOMA-IR, homeostasis model assessment for insulin resistance; LDH, lactate dehydrogenase; $\mathrm{NH}_{3}$, ammonia; TCho, total cholesterol; TG, triglyceride; wk, weeks. Results are shown as means $\pm \mathrm{SE}$. BCAA,$- n=9$; $\mathrm{BCAA}+, n=9 .{ }^{*} p<0.05$ between 0 and $12 \mathrm{wk}$ determined by paired $t$-test.

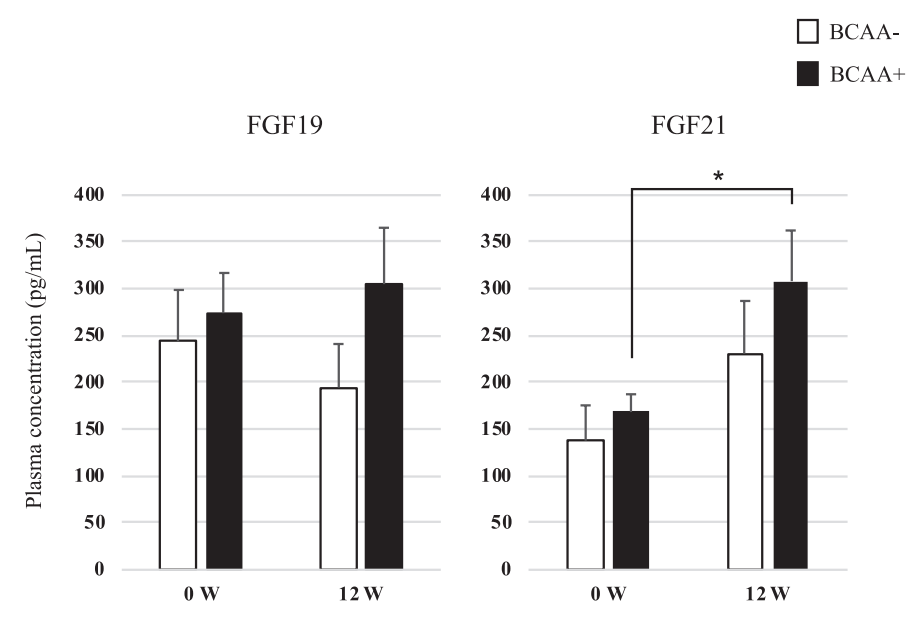

Fig. 1. Plasma concentrations of FGF19 and FGF21. Plasma levels of FGF21 increased during glucocorticoid tapering and was significantly larger in BCAA+ patients.

the past 6 mo, cumulative dose during the study, and dose at the beginning and end of the study. Notably, the daily GC dose decreased significantly between the start and end of the study, from $17.9 \pm 7.5$ to $13.5 \pm 3.4(p<$ $0.05)$ and $18.0 \pm 4.8$ to $13.9 \pm 3.3 \mathrm{mg}(p<0.05)$ for both the $\mathrm{BCAA}-$ and $\mathrm{BCAA}+$ groups, respectively, at the start and end of the study. The decrease in GC dose was similar between the two groups (Supplemental Online Material, Table S1) (7). Muscle CSA in the biceps femoris muscle improved significantly after $12 \mathrm{wk}$ in both groups. In addition, greater improvements were observed in the biceps femoris muscle in the $\mathrm{BCAA}+$ group than in the BCAA - group (Supplemental Online Material, Table S2) (7).

\section{Changes in biochemical parameters}

The influence of BCAA supplementation on various biochemical and metabolic parameters was investigated. As summarized in Table 1, liver function was comparable between BCAA - and BCAA + patients after supplementation. In contrast, creatinine $(\mathrm{Cr})$ was significantly elevated in $\mathrm{BCAA}+$ patients. Since those patients did not have apparent kidney involvement during the study, $\mathrm{Cr}$ elevation might represent the increase in skeletal muscle mass (10). Moreover, 
Table 2. Plasma amino acid concentrations of the participants.

\begin{tabular}{|c|c|c|c|c|c|c|c|}
\hline & \multirow{2}{*}{$\begin{array}{l}\text { Standard values } \\
\quad(\mathrm{nmol} / \mathrm{mL})\end{array}$} & \multicolumn{3}{|c|}{ BCAA- } & \multicolumn{3}{|c|}{$\mathrm{BCAA}+$} \\
\hline & & $0 \mathrm{wk}$ & $12 \mathrm{wk}$ & $p$-Value & $0 \mathrm{wk}$ & $12 \mathrm{wk}$ & $p$-Value \\
\hline Isoleucine & $43.0-112.8$ & $73.5 \pm 6.1$ & $58.0 \pm 2.9$ & $0.015^{*}$ & $67.8 \pm 4.7$ & $57.8 \pm 3.2$ & 0.147 \\
\hline Leucine & $76.6-171.3$ & $130.6 \pm 7.4$ & $110.1 \pm 4.8$ & $0.001^{*}$ & $124.3 \pm 7.6$ & $111.3 \pm 3.8$ & 0.166 \\
\hline Valine & $147.8-307.0$ & $239.1 \pm 12.9$ & $204.9 \pm 11.1$ & $0.004^{*}$ & $225.6 \pm 13.5$ & $210.2 \pm 10.0$ & 0.405 \\
\hline Alanine & $208.7-522.7$ & $421.0 \pm 29.0$ & $371.0 \pm 26.9$ & 0.212 & $408.3 \pm 28.5$ & $378.5 \pm 31.7$ & 0.292 \\
\hline Aspartate & $<2.4$ & $2.7 \pm 0.6$ & $1.7 \pm 0.4$ & 0.120 & $3.6 \pm 0.4$ & $2.6 \pm 0.3$ & $0.026^{*}$ \\
\hline Glutamate & $12.6-62.5$ & $37.5 \pm 5.3$ & $34.1 \pm 2.3$ & 0.478 & $59.6 \pm 6.6$ & $49.1 \pm 6.1$ & 0.202 \\
\hline Glutamine & $422.1-703.8$ & $547.1 \pm 21.8$ & $554.9 \pm 21.7$ & 0.676 & $522.1 \pm 22.4$ & $529.0 \pm 15.7$ & 0.724 \\
\hline TAA & $2,068.2-3,510.3$ & $2,836.9 \pm 1,34.0$ & $2,551.0 \pm 77.7$ & 0.052 & $2,842.8 \pm 114.0$ & $2,669.0 \pm 75.1$ & 0.097 \\
\hline NEAA & 1,381.6-2,379.4 & $1,852.9 \pm 87.5$ & $1,701.3 \pm 55.6$ & 0.124 & $1,891.9 \pm 76.8$ & $1,789.9 \pm 61.6$ & $0.045^{*}$ \\
\hline EAA & $660.0-1,222.3$ & $984.0 \pm 53.8$ & $849.7 \pm 34.6$ & $0.022^{*}$ & $950.9 \pm 42.0$ & $879.1 \pm 33.5$ & 0.273 \\
\hline BCAA & $265.8-579.1$ & $443.2 \pm 26.1$ & $373.1 \pm 18.0$ & $0.004^{*}$ & $417.7 \pm 25.2$ & $379.3 \pm 16.1$ & 0.253 \\
\hline
\end{tabular}

Instead of evaluation of cystein, the concentrarion of cystine is depicted. BCAA, branched chain amino acids; EAA, essential amino acids; NEAA, non-essential amino acids; TAA, total amino acids; wk, weeks. Results are shown as means \pm SE. BCAA,$- n=9$; BCAA,$+ n=9 .{ }^{*} p<0.05$ between 0 and $12 \mathrm{wk}$ determined by paired $t$-test. ${ }^{\dagger} p<0.05$ between BCAA - and $\mathrm{BCAA}+$ at 0 and $12 \mathrm{wk}$ determined by unpaired $t$-test.

Table 3. Urine amino acid and 3-methylhistidine concentrations of the participants.

\begin{tabular}{|c|c|c|c|c|c|c|}
\hline \multirow{2}{*}{$\mathrm{nmol} / \mathrm{mgCRE}$} & \multicolumn{3}{|c|}{$\mathrm{BCAA-}$} & \multicolumn{3}{|c|}{$\mathrm{BCAA}+$} \\
\hline & $0 \mathrm{wk}$ & $12 \mathrm{wk}$ & $p$-Value & $0 \mathrm{wk}$ & $12 \mathrm{wk}$ & $p$-Value \\
\hline Isoleucine & $18.6 \pm 2.7$ & $10.7 \pm 0.5$ & $0.013^{*}$ & $18.1 \pm 1.1$ & $12.9 \pm 0.9$ & $0.002 *$ \\
\hline Leucine & $49.1 \pm 5.9$ & $35.6 \pm 6.4$ & $0.029 *$ & $43.3 \pm 4.8$ & $29.6 \pm 3.8$ & $0.008^{*}$ \\
\hline Valine & $45.6 \pm 5.5$ & $35.2 \pm 5.0$ & $0.014^{*}$ & $37.5 \pm 4.7$ & $32.8 \pm 3.0$ & 0.102 \\
\hline 3-Methylhistidine & $228.9 \pm 18.0$ & $191.2 \pm 14.9$ & 0.098 & $262.2 \pm 12.5$ & $181.2 \pm 11.2$ & $0.001^{*}$ \\
\hline
\end{tabular}

Results are corrected with urine creatinine concentration (nmol/mgCRE) and shown as means \pm SE. BCAA,$- n=9$; $\mathrm{BCAA}+$, $n=9 .{ }^{*} p<0.05$ between 0 and 12 wk determined by paired $t$-test.

$\mathrm{BCAA}+$ patients exhibited an increase in albumin and a decrease in ammonia $\left(\mathrm{NH}_{3}\right)$, indicating an increase in protein synthesis and a decrease in protein catabolism by BCAA, respectively $(11,12)$. Urinary secretion of 3-methylhistidine $(\mathrm{MH})$ was significantly decreased in $\mathrm{BCAA}+$ patients, suggesting a decrease in muscle degradation (13). Metabolic parameters including levels of plasma glucose, insulin, triglyceride, free fatty acids, hemoglobin A1c, and homeostasis model assessment of insulin resistance (HOMA-IR) were comparable between $\mathrm{BCAA}-$ and $\mathrm{BCAA}+$ patients.

We and others have reported on the role of the FGF family members FGF19 and FGF21 in the regulation of skeletal muscle mass $(6,14)$. In our study, plasma levels of FGF21 were elevated along with tapering GC, and such elevation was larger in BCAA + patients (Fig. 1). Changes in plasma and urinary amino acid concentrations

In the present study, the amino acids related to BCAA metabolism were measured. Table 2 shows the changes in plasma BCAA and amino acid concentrations related to muscle metabolic responses, and Table 3 shows changes in urinary BCAA and 3-MH reflecting muscle catabolism. Plasma BCAA concentrations decreased, especially in the BCAA - group, which showed a signifi-

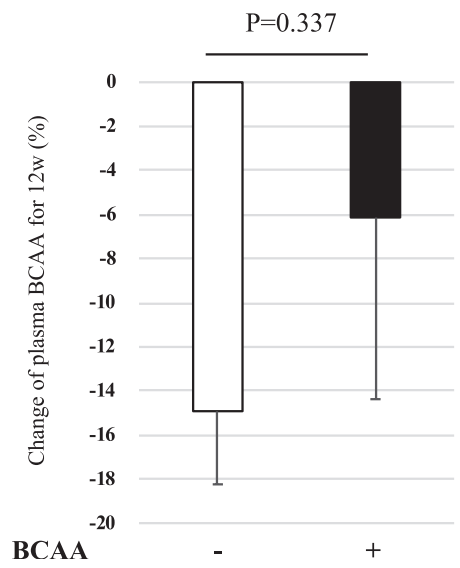

Fig. 2. Changes in plasma branched-chain amino acid concentrations. Plasma BCAA concentrations decreased in both groups, but the decrease in BCAA- patients was less than that in patients treated with BCAA.

cant decrease. Plasma alanine, aspartate and glutamate concentrations (15), in which BCAA acts as a nitrogen donor, were also decreased in both groups. The plasma aspartate concentration decreased significantly in the 
A

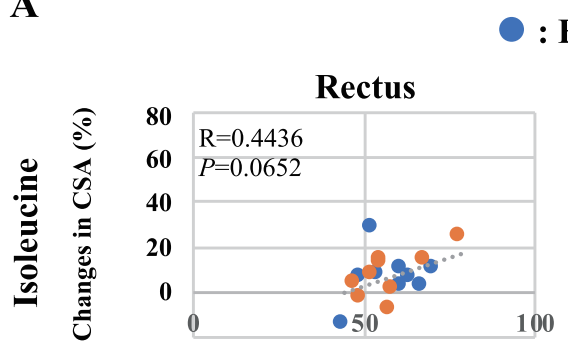

\section{: BCAA-}
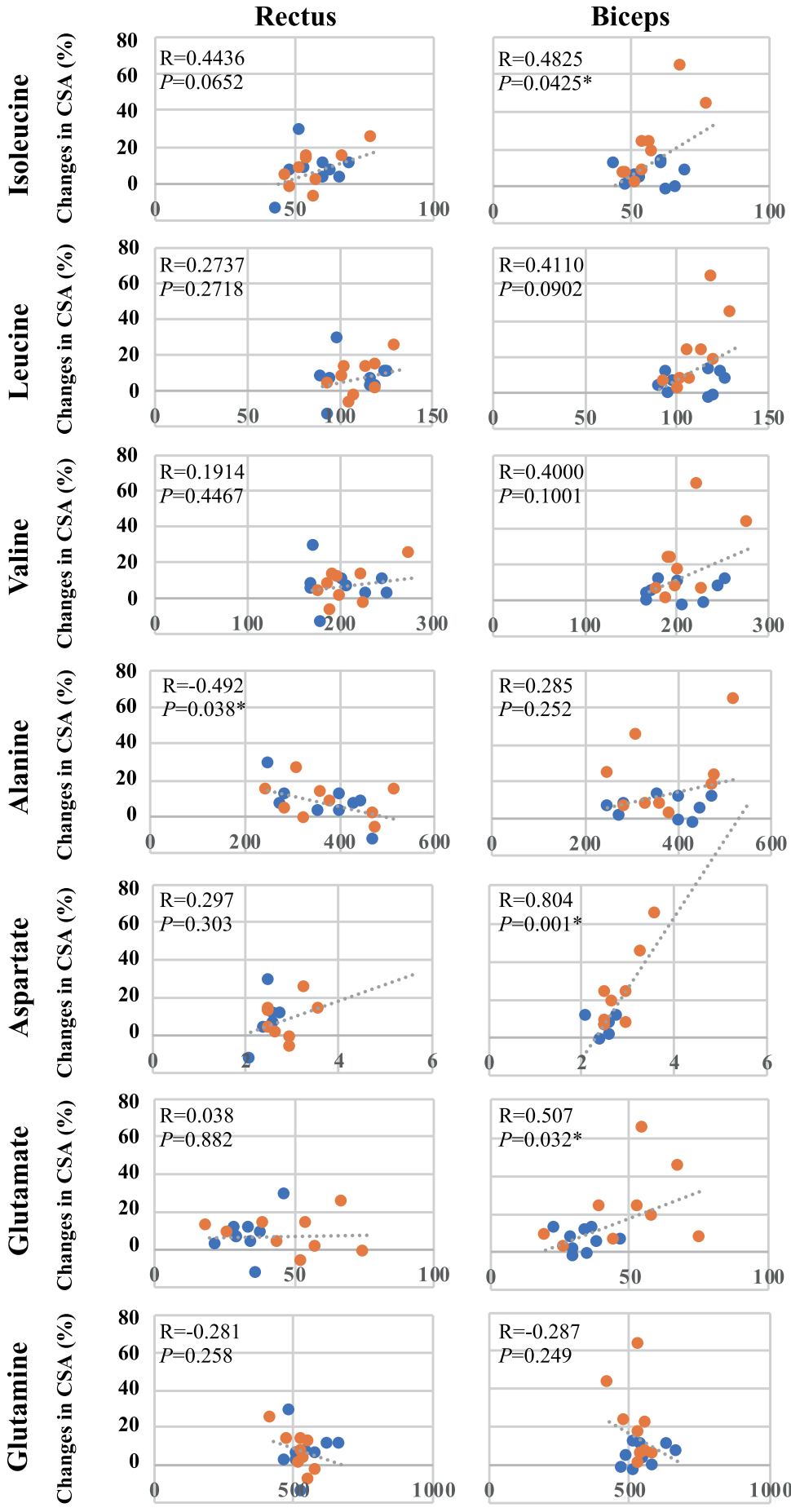

Amino acids concentration $(\mathrm{nmol} / \mathrm{mL})$

Amino acids concentration $(\mathrm{nmol} / \mathrm{mL})$

Fig. 3

$\mathrm{BCAA}+$ group. Plasma glutamine concentrations were unchanged. Both groups showed a decrease in total amino acid concentration, with non-essential amino acid concentrations lower in the BCAA+ group and essential amino acid concentrations lower in the BCAA - group. Plasma BCAA concentrations decreased in both groups, but the decrease in in BCAA+ group was less than that of the BCAA - group (Fig. 2). Urinary isoleucine and leucine concentrations decreased in both groups, and the urinary secretion of 3-MH was reduced in the BCAA + group, suggesting reduced muscle degradation (Table 3) (13). 
B

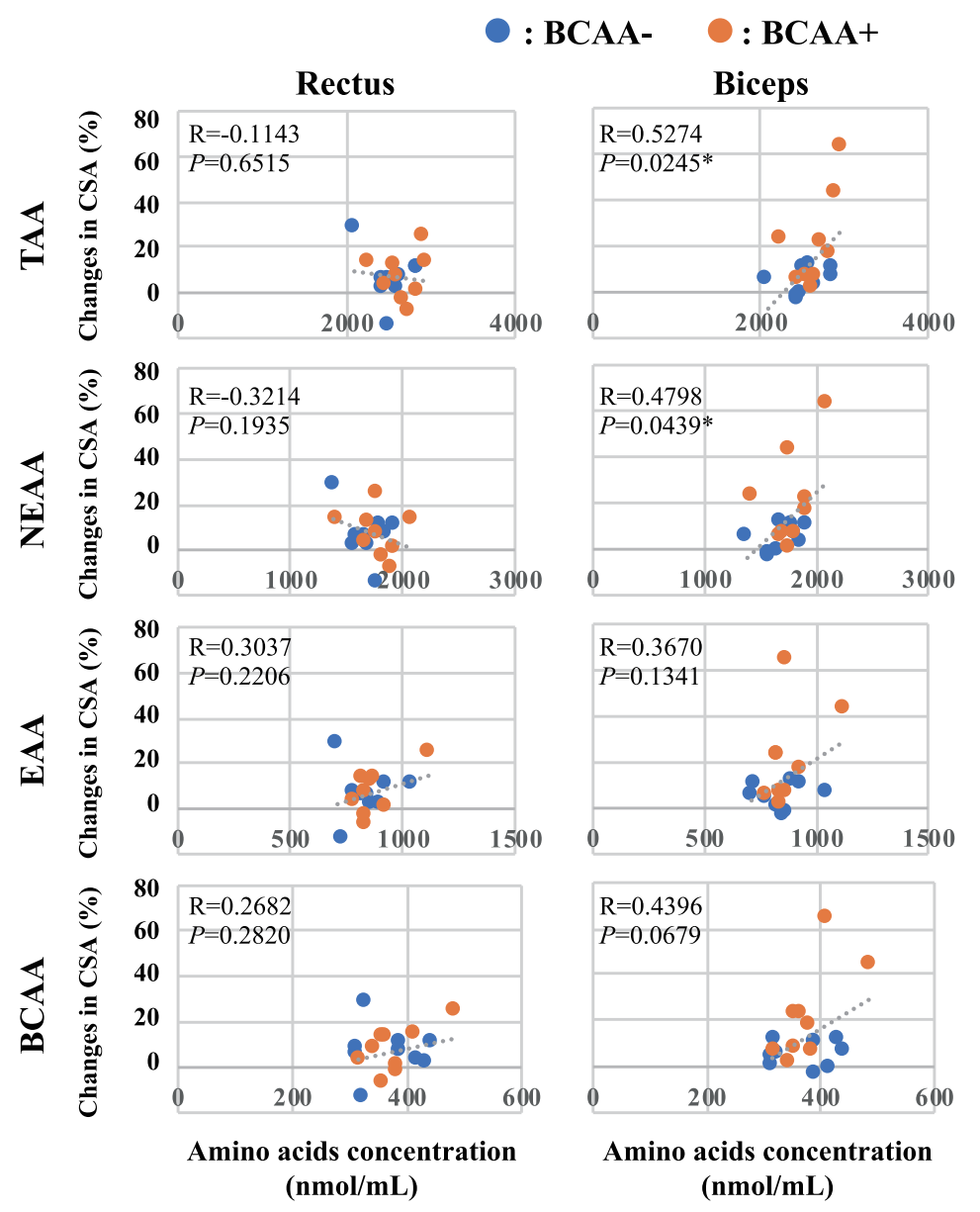

Fig. 3. Relationship between plasma amino acid concentration at $12 \mathrm{wk}$ and muscle CSA change ratio. A significant positive correlation was observed between plasma isoleucine, aspartate, and glutamate concentrations at 12 wk and improvement in muscle atrophy in the biceps femoris muscle. In contrast, no such correlation was found in the rectus femoris muscle (A). A significant positive correlation was also observed between the total amino acid and non-essential amino acid concentrations and the rate of improvement in biceps femoris at $12 \mathrm{wk}$. We also observed a trend of toward a positive correlation between BCAA concentration and the rate of improvement in the biceps femoris (B).

Association between plasma amino acid concentration and rate of change in muscle CSA at $12 \mathrm{wk}$

As shown in Fig. 3A, a significant positive correlation was observed between plasma isoleucine concentrations at $12 \mathrm{wk}$ and improvement in muscle atrophy in the biceps femoris. Leucine and valine also showed a tendency of such positive correlation. In contrast, no significant correlation was found in the rectus femoris in BCAAs. Plasma alanine concentration showed a negative correlation with improvement in muscle atrophy in the rectus femoris, while this correlation was significantly positive for aspartate and glutamate in the biceps femoris. Glutamate concentration also correlated positively with improvement in muscle atrophy in the biceps femoris muscle. A positive correlation was observed between total and non-essential amino acid concentration and the rate of improvement in the biceps femoris at $12 \mathrm{wk}$. A trend of positive correlation was observed between BCAA concentration and the rate of improvement in the biceps femoris (Fig. 3B).

\section{DISCUSSION}

As expected, variations in the effects of BCAA supplementation on biochemical and metabolic parameters were observed, suggesting that muscle protein synthesis increased and its degradation decreased. Cr, albumin, $\mathrm{NH}_{3}$, and urinary 3-MH levels were used as markers reflecting catabolism and anabolism, even during GC treatment. FGF19 which is the rate-limiting enzyme of bile acid synthesis, is reported to ameliorated skeletal muscle atrophy induced by GC treatment and obesity in mice (16). However, in the present study, no such changes were observed. This difference in results may be due to the stronger effect of the administered GC. In the same FGF subfamily, FGF21 acts on organs to induce a variety of changes, including the browning of white adipose tissue, suppression of triglyceride accumulation in the liver, and increased insulin sensitivity in muscle, a marker of energy metabolism $(17,18)$. Interestingly, FGF21 levels increased with GC tapering. FGF21 might be useful as a marker to improve the side 
effects of GC metabolism.

Free amino acids in plasma serve as important hub substances in human metabolism. They are transported between organs and tissues through the blood, and their concentrations in plasma are strictly controlled by the homeostatic mechanisms of the organs. However, plasma amino acid concentrations may fluctuate depending on the timing of blood sampling and subsequent storage conditions. In the present study, blood samples were collected after fasting, and plasma was promptly separated from whole blood and frozen for storage to minimize the effects of amino-acid-metabolizing enzymes (19). The BCAA extracted as a useful marker in this study is an essential amino acid. Since the blood was collected during fasting, the plasma BCAA concentration can be considered as the trough concentration of the subject. This homeostasis is disrupted by certain diseases and treatments. For example, it is well known that in cirrhosis, the ratio of BCAA to aromatic amino acids (Fisher's ratio) is reduced (20). The presence of chronic insulin resistance has been reported to promote BCAA oxidation in skeletal muscle (21). The concentrations of some amino acids are altered in malignancies $(22,23)$ and inflammatory diseases (24). Although the underlying diseases were diverse in the subject cohort of this study, the diseases were very well controlled by GC, and patients with malignancies were excluded. Thus, this study cohort was ideal for studying GC-induced myopathy.

Only a few studies have examined the effect of GC treatment on plasma amino acid concentrations, and these have all investigated short-term GC administration. The administration of $60 \mathrm{mg} / \mathrm{d}$ of prednisolone to healthy subjects for $3 \mathrm{~d}$ is reported to increase the concentration of most amino acids, including BCAA, and it was concluded that this effect was caused by increased muscle proteolysis by GC (25). However, no study had reported on GC administration for a prolonged period, as in the present study. Thus this study is the first such report and provides valuable and important data for practice against sarcopenia.

In this study of long-term GC treatment, we observed that plasma BCAA concentrations in both the BCAAand $\mathrm{BCAA}+$ groups were within reference values at $12 \mathrm{wk}$ into the study. However, we noted a tendency for the concentrations of total plasma amino acids, and non-essential and essential amino acids, including BCAA, to decrease over the course of the study. In particular, plasma BCAA concentrations significantly decreased in the BCAA - group. There was no report concerned with amino acid profile in the patients treated with long-term GC treatments, but Cushing's disease is similar condition to the long-term GC treatments. Plasma BCAA concentrations are reported to be reduced in Cushing's disease (26).

Our biochemical data indicate that the BCAA + group was most likely to have lower plasma concentrations due to the uptake of plasma BCAA into the muscle for muscle protein synthesis. In addition to the effects of disorders, there may be physiological effects due to GC.
A study in rats reports that the BCAA excretion transporter $\mathrm{y}+\mathrm{LAT} 1$ (SLC7A7) expressed in the kidney is upregulated by GC, resulting in increased urinary BCAA concentrations (27). Although there still have been no report on the renal BCAA excretion transporter in humans, an average decrease in GC of $4 \mathrm{mg} / \mathrm{d}$ during the study period might be suggested to have reduced $\mathrm{y}+$ LAT1 (SLC7A7) expression and decreased urinary BCAA concentrations, albeit slightly. Since GC treatment in rheumatic diseases usually lasts for more than a few years, taking measures to address these decreases in plasma amino acid concentrations is important.

In our previous study, we found that the effect of BCAA administration on GC-induced myopathy was stronger in the biceps femoris than in the rectus femoris (7). This observation may be due to the high expression of GC receptors in the rectus femoris, a fast-twitch muscle. In response to GC, GC receptors activate muscle-atrophy-related genes to offset the effects of BCAA. For this reason, the effects of BCAA are more likely to be reflected in the biceps femoris, which expresses fewer GC receptors. We also found that plasma amino acid concentrations at $12 \mathrm{wk}$ correlated positively with improvement in atrophied muscle. However, individual differences were observed in the level of improvement in muscle atrophy between the BCAA + group and the $\mathrm{BCAA}$ - group. Regardless of BCAA administration, there were cases in which low plasma amino acid concentrations and little improvement in muscle atrophy (Fig. 2). These results suggest that one of the factors contributing to the decrease in muscle atrophy may be the concentration of amino acids in the plasma. These findings suggest that plasma BCAA, aspartate (28), and glutamate $(29,30)$ levels may also reflect improvement of muscle atrophy in skeletal muscle, especially in slowtwitch muscle. In skeletal muscle, glutamate is biosynthesized from BCAAs, which may only reflect the muscle volume increase by BCAAs, but muscle atrophy is further improved when BCAAs are prescribed with glutamine, which is produced from glutamate and ammonia in the urea cycle (31). In addition, aspartate stimulates ATP synthesis in the purine nucleotide cycle and suppresses slow twitch muscle atrophy (32). These suggest that plasma glutamate and aspartate, as well as BCAA, may be involved in the improvement of muscle atrophy.

The present results indicate that BCAA supplementation is useful in improving muscle atrophy in slowtwitch muscle during GC treatment. Because BCAA are less effective in fast-twitch muscles while on GC, a combination of anaerobic exercise may be recommended to improve fast-twitch muscles. However, devising a training menu tailored to their condition and performance status is needed because of many elderly patients.

Recently, it was also reported that GC-pulse therapy does not advance muscle atrophy over daily GC administration (33). Future studies are needed to validate GC delivery methods and especially develop drugs for improving fast twitch muscles.

This study has several limitations. Because this inves- 
tigation is a pilot study, the number of patients in the cohort is small and unblinded. Therefore, it is necessary to further increase the number of cases to verify the results of significant differences in this study. GC administration differed between patients. In future studies, the number of subjects needs to be higher and GC administration should be controlled. We consider that the findings of this study are important for clinical practice against sarcopenia, and it will contribute to the development of new monitoring and treatment strategies for steroid-induced muscular atrophy. Adjusting BCAA doses according to plasma BCAA concentrations and the rehabilitation of fast-twitch muscles could contribute to the possible prevention of GC-induced myopathy.

\section{CONCLUSION}

Plasma amino acid levels decreased substantially during GC administration, and in particular, isoleucine, aspartate, and glutamate concentrations were shown to be potentially useful markers of improvement in slow twitch muscle atrophy during BCAA treatment.

\section{Authorship}

NY, MY, and HT were involved in the conception and design of the study, analysis and interpretation of data, and drafting of the manuscript. AK, MU, and HY were involved in the acquisition and analysis of data. All authors read the manuscript critically, provided comments, and approved the final version.

NY and MY contributed equally to this work.

\section{Disclosure of state of COI}

No conflicts of interest to be declared.

\section{Acknowledgments}

We acknowledge everyone who contributes to our study. Our work was supported by JSPS KAKENHI Grant Numbers JP16H05330 and JP18KT0017 to H.T., JP16K09230 to N.Y., JP17K16158 and JP20K17528 to H.Y., by AMED Grant "Project for Whole Implementation to Support and Ensure the Female Life"

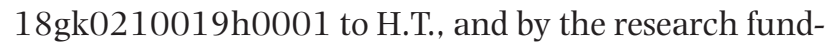
ing in IMSUT Hospital, the Institute of Medical Science, the University of Tokyo. These fund had no control over the interpretation, writing, or publication of this work.

\section{Supporting information}

Supplemental online material is available on J-STAGE.

\section{REFERENCES}

1) Mukund K, Subramaniam S. 2020. Skeletal muscle: a revier of molecular structure and function, in health and disease. Wiley Interdiscip Rev Syst Biol Med 12: e1462.

2) Cruz-Jentoft AJ, Sayer AA. 2019. Sarcopenia. Lancet 393: 2636-2646.

3) Petta I, Peene I, Elewaut D, Vereecke L, Bosscher K. 2019. Risks and benefits of corticosteroids in arthritic diseases in the clinic. Biochem Pharmacol 165: 112125.

4) Martín AI, Priego T, López-Calderón A. 2018. Hor- mones and muscle atrophy. Adv Exp Med Biol 1088: 207-233.

5) Shimizu N, Yoshikawa N, Ito N, Maruyama T, Suzuki Y, Takeda S, Nakae J, Tagata Y, Nishitani S, Takehara K, Sano M, Fukuda K, Suematsu M, Morimoto C, Tanaka H. 2011. Crosstalk between glucocorticoid receptor and nutritional sensor mTOR in skeletal muscle. Cell Metab 13: 170-182.

6) Shimizu N, Maruyama T, Yoshikawa N, Matsumiya R, Ma Y, Ito N, Tasaka Y, Kuribara-Souta A, Miyata K, Oike Y, Berger S, Schütz G, Takeda S, Tanaka H. 2015. A muscle-liver-fat signalling axis is essential for central control of adaptive adipose remodeling. Nat Commun $\mathbf{6}$ : 6693.

7) Yoshikawa N, Shimizu N, Uehara M, Oda A, Matsumiya R, Matsubara E, Kobayashi H, Hosono O, Kuribara-Souta A, Baba H, Nagamura F, Kiryu S, Tanaka H. 2017. The effects of bolus supplementation of branched-chain amino acids on skeletal muscle mass, strength, and function in patients with rheumatic disorders during glucocorticoid treatment. Mod Rheumatol 27: 508-517.

8) Hosono O, Yoshikawa N, Shimizu N, Kiryu S, Uehara M, Kobayashi H, Matsumiya R, Kuribara A, Maruyama T, Tanaka H. 2015. Quantitative analysis of skeletal muscle mass in patients with rheumatic diseases under glucocorticoid therapy-comparison among bioelectrical impedance analysis, computed tomography, and magnetic resonance imaging. Mod Rheumatol 25: 257-263.

9) Schneider CA, Rasband WS, Eliceiri KW. 2012. NIH Image to Imagej: 25 years of image analysis. Nat Methods 9: 671-675.

10) Schutte JE, Longhurst JC, Gaffney FA, Bastian BC, Blomqvist CG. 1981. Total plasma creatinine: an accurate measure of total striated muscle mass. J Appl Physiol Respir Environ Exerc Physiol 51: 762-766.

11) Baumgartner RN, Koehler KM, Garry LRP. 1996. Serum albumin is associated with skeletal muscle in elderly men and women. Am J Clin Nutr 64: 552-558.

12) Graham TE, MacLean DA. 1998. Ammonia and amino acid metabolism in skeletal muscle: human, rodent and canine models. Med Sci Sports Exerc 30: 34-46.

13) Chinkes DL. 2005. Methods for measuring tissue protein breakdown rate in vivo. Curr Opin Clin Nutr Metab Care 8: 534-537.

14) Tezze C. 2017. Age-associated loss of OPA1 in muscle impacts muscle mass, metabolic homeostasis, systemic inflammation, and epithelial senescence. Cell Metab 25: 1374-1389.

15) Harper AE, Miller RH, Block KP. 1984. Branched-chain amino acid metabolism. Annu Rev Nutr 4: 409-454.

16) Benoit B, Meugnier E, Castelli M, Chanon S, Vieille-Marchiset A, Durand C, Bendridi N, Pesenti S, Monternier P-A, Durieux A-C, Freyssenet D, Rieusset J, Lefai E, Vidal H, Ruzzin J. 2017. Fibroblast growth factor 19 regulates skeletal muscle mass and ameliorates muscle wasting in mice. Nat Med 23: 990-996.

17) Rydén M. 2009. Fibroblast growth factor 21: an overview from a clinical perspective. Cell Mol Life Sci 66: 2067-2073.

18) Kharitonenkov A, Wroblewski VJ, Koester A, Chen Y-F, Clutinger CK, Tigno XT, Hansen BC, Shanafelt AB, Etgen GJ. 2007. The metabolic state of diabetic monkeys is regulated by fibroblast growth factor-21. Endocrinology 148: 774-781. 
19) Takehara S, Yoshida H, Ozawa S, Yamazaki J, Shimbo K, Nakayama A, Mizukoshi T, Miyano H. 2016. The effects of pre-analysis sample handling on human plasma amino acid concentrations. Clin Chem Acta 455: 68-74.

20) Chang ML, Yang SS. 2019. Metabolic signature of hepatic fibrosis: from individual pathways to system biology. Cells 8: 1423.

21) Neinast MD, Jang C, Hui S, Murashige DS, Chu Q, Morscher RJ, Li X, Zhan L, White E, Anthony TG, Rabinowitz JD, Arany Z. 2019. Quantitative analysis of the whole-body metabolic fate of branched-chain amino acids. Cell Metab 29: 417-429.e4.

22) Katagiri R, Goto A, Nakagawa T, Nishiumi S, Kobayashi T, Hidaka A, Budhathoki S, Yamaji T, Sawada N, Shimazu T, Inoue M, Iwasaki M, Yoshida M, Tsugane S. 2018. Increased levels of branched-chain amino acid associated with increased risk of pancreatic cancer in a prospective case-control study of a large cohort. Gastroenterology 155: 1474-1482.e1.

23) Wang X, Wang J, Wang Z, Wang Q, Li H. 2015. Dynamic monitoring of plasma amino acids and carnitine during chemotherapy of patients with alimentary canal malignancies and its clinical value. Onco Targets Ther 8: 1989-1996.

24) Trang LE, Früst T, Odebäck AC, Lövgren O. 1985. Plasma amino acids in rheumatoid arthritis. Scand J Rheumatol 14: 393-402.

25) Löfberg E, Gutierrez A, Wererman J, Anderstam B, Mitch WE, Price SR, Bergström J, Alvestrand A. 2002. Effects of high dose of glucocorticoids on free amino acids, ribosomes and protein turnover in human muscle. Eur J Clin Invest 32: 345-353.

26) Faggiano A, Pivonello R, Melis D, Alfieri R, Filippella M,
Spagnuolo G, Salvatore F, Lombardi G, Colao A. 2002. Evaluation of circulating levels and renal clearance of natural amino acids in patients with Cushing's disease. Endocrinol Invest 25: 142-151.

27) Schwertfeger M, Pissowotzki K, Fleck Ch, Taylor PM. 2003. Regulation of L-leucine transport in rat kidney by dexamethasone and triiodothyronine. Amino Acids 25: 75-83.

28) Liu Y, Wang X, Leng W, Pi D, Tu Z, Zhu H, Shi H, Li S, Hou Y, Hu C-AA. 2017. Aspartate inhibits LPS-induced MAFbx and MuRF1 expression in skeletal muscle in weaned pigs by regulating Akt, AMPK $\alpha$ and FOXO1. Innate Immun 23: 34-43.

29) Turinsky J, Long CL. 1990. Free amino acids in muscle: effect of muscle fiber population and denervation. Am J Physiol 258: E485-491.

30) Rutten EPA, Engelen MPKJ, Schols AMWJ, Deutz NEP. 2005. Skeletal muscle glutamate metabolism in health and disease: state of the art. Curr Opin Clin Nutr Metab Care 8: 41-51.

31) Haba Y, Fujimura T, Oyama K, Kinoshita J, Miyashita T, Fushida A, Harada S, Ohta T. 2019. Effect of oral branched-chain amino acids and glutamine supplementation on skeletal muscle atrophy after total gastrectomy in rat model. J Surg Res 243: 281-288.

32) Yamada S, Kizaki E, Ozeki A, Nakagawa M, Fujita H. 2015. Sarcopenia and aspartic acid magnesium. J Phys Fitness Sport Med 4: 287-294.

33) Quattrocelli M, Zelikovich AS, Jiang Z, Peek CB, Demonbreun AR, Kuntz NL, Barish GD, Haldar SM, Bass J, McNally EM. 2019. Pulsed glucocorticoids enhance dystrophic muscle performance through epigenetic metabolic reprogramming. JCI Insight 4: e132402. 